\title{
Review of Medical Advisory Services by the Korean Society of Pathologists from 2003 to 2014
}

\begin{abstract}
Min Hye Jang · Geon Kook Lee ${ }^{1}$ Han Seong Kim² . Wan Seop Kim

Department of Pathology, Konkuk University School of Medicine, Seoul; ' ${ }^{1}$ Department of Pathology, National Cancer Center, Goyang; ${ }^{2}$ Department of Pathology, Inje University College of Medicine, Goyang, Korea
\end{abstract}

Received: September 14, 2015

Accepted: September 17, 2015

Corresponding Author

Wan Seop Kim, MD, PhD

Department of Pathology, Konkuk University

School of Medicine, 120 Neungdong-ro,

Gwangiin-gu, Seoul 05029, Korea

Tel: +82-2-2030-5642

Fax: +82-2-2030-5629

E-mail:wskim@kuh.ac.kr

\begin{abstract}
Background: Since 2003, the Korean Society of Pathologists (KSP) has been officially providing medical advisory services (MAS). We reviewed the cases submitted to the KSP between 2003 and 2014. Methods: In total, 1,950 cases were submitted, most by private health insurance companies. The main purposes of the consultations were to clarify the initial diagnoses and to assign a proper disease classification code. We comprehensively reviewed 1,803 consultation cases with detailed information. Results: In spite of some fluctuations, the number of submitted cases has been significantly increasing over the 12 study years. The colon and rectum (40.3\%), urinary bladder (14.2\%), and stomach (6.9\%) were the three most common tissues of origin. The most common diagnoses for each of the three tissues of origin were neuroendocrine tumor $(50.7 \%)$, non-invasive papillary urothelial carcinoma (70.7\%), and adenocarcinoma (36.2\%). Regardless of the tissue of origin, neuroendocrine tumor of the digestive system was the most common diagnosis (419 of 1,803). Conclusions: In the current study, we found that pathologic consultations associated with private health insurance accounted for a large proportion of the MAS. Coding of the biologic behavior of diseases was the main issue of the consultations. In spite of the effort of the KSP to set proper guidelines for coding and classification of tumors, this review revealed that problems still exist and will continue to be an important issue.
\end{abstract}

Key Words: Medical advisory service; Pathologic consultation; Private health insurance
Advisory services are widely used in the field of medicine in many countries. Doctors commonly use consultations, especially in surgical pathology practice. In the United States, the Association of Directors of Anatomic and Surgical Pathology has defined various kinds of pathology consultations and devised regulations to control the quality of each type. ${ }^{1-3}$

In Korea, pathologic consultations by doctors have also been performed in daily practice. However, most of these consultations have been conducted informally, without regulations or proper fees. Therefore, Korean pathologists agreed with the necessity for an official pathology advisory service system and organized the Medical Advisory Committee (MAC) as a subdivision of the Korean Society of Pathologists (KSP) in 2003. The $\mathrm{MAC}$ has been officially providing various kinds of advisory services, focusing on diagnostic pathology, since 2003. They have restrained the extent of users and contexts of pathologic consultations and have established detailed regulations. According to these regulations, certain individuals or institutions can use the consultation services: health and medical institutions run by government agencies such as the Ministry of Health and Welfare or community health centers; investigative authorities such as de- partments of prosecution or police departments; judicial offices; the Korean Medical Association; private insurance companies; specific institutions or individuals who are allowed to use the services of the KSP; and the members of the KSP.

The contents of consultations are defined by the third provision of the regulations of the medical advisory services (MAS): classification and evaluation of the grade of malignant or premalignant lesion; confirmation of a pathologic diagnosis, including review of slides or pathologic reports; pathologic review of controversial cases among pathologists or clinicians; consultation about rare diseases; and other medical questions allowed by the MAC.

The consultation process is quite simple (Fig. 1). After a client requests a pathologic consultation with the KSP, the advisory manager who is appointed by the MAC reviews the contents of the consultation. Then, he or she designates a specific pathologist as a consultant, considering his or her experience and subspecialty in pathologic fields. The appointed pathologist must have more than 10 years of experience as a surgical pathologist of the specific subspecialty. There must be no conflicts of interest between the consultant and client. After reviewing the consult- 


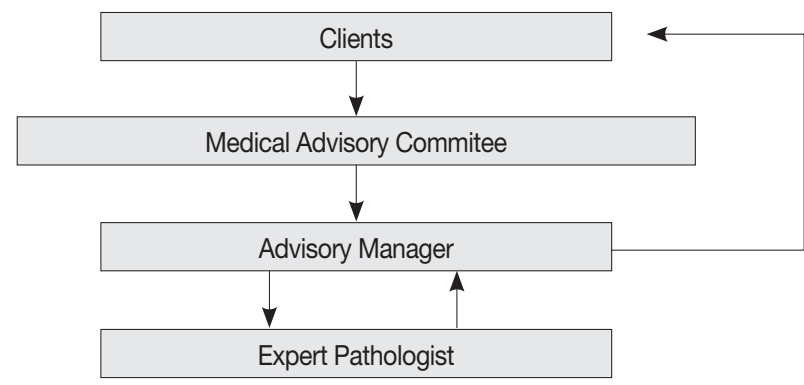

Fig. 1. Schematic flow chart of the medical advisory service system of the Korean Society of Pathology (KSP).

ed case, the assigned pathologist should report the result to the advisory manager within 2 weeks. Then, the advisory manager informs the clients of the result.

In this review, we discuss the medical advisory service system of Korea provided by the KSP and comprehensively review the consultation cases from 2003 to 2014.

\section{MATERIALS AND METHODS}

The consultation cases are stored in a web-based medical advisory service system (http://jamoon.pathology.or.kr) that revealed 1,950 cases sent to the MAC from 2003 to 2014 (Fig. 2). We obtained the following specific information for each case: person who submitted the case, date on which the case was sent, assigned consulting pathologist, tissue of origin for the submitted case, and final diagnosis. After 46 cases with missing data were excluded from the review, 1,904 cases were reviewed and analyzed for this study. This study was approved by the Institutional Review Board of Konkuk University Hospital (KUH1210045).

Although various institutions and individuals were allowed to use the services, all but nine consultations were requested by private health insurance companies or insurance adjustment companies. The other nine cases were submitted by district courts or legal agencies.

The questions asked about the submitted cases were also limited. The major contents of the consultations were the same in most of the cases: "What is the precise pathologic diagnosis of the patient's illness?" and "Which classification code should be given considering the biologic nature and behavior of the illness?" To obtain answers to these questions, the submitters asked that the surgical pathology or cytopathology slides and original pathologic reports be reviewed, and that the consulting pathologist clarify the degree of malignancy using an accurate disease classification code based on the International Classification of Diseases for Oncology (ICD-O) $)^{4}$ or the Korean Classifi- cation of Diseases (KCD). ${ }^{5}$

\section{RESULTS}

\section{Number of consultation cases and distribution of tissues of origin}

The number of submitted cases between 2003 and 2014 is presented in Fig. 2A. Although there was some fluctuation between 2007 and 2013, the number of submitted cases has been significantly increasing over the 12 study years, from four cases in 2003 to 296 cases in 2014.

We analyzed the tissues of origin of 1,904 cases from 2003 to 2014. The distributions of tissues of origin were slightly different throughout the years (Fig. 2A, Electronic Supplementary Table S1). The three most common tissues of origin from 2003 to 2014 were the colon and rectum $(767,40.3 \%)$, the urinary bladder $(271,14.2 \%)$, and the stomach $(132,6.9 \%)$. Cases with these three tissues of origin comprised more than half of the entire set of submitted cases. The next most common tissues of origin included the breast, ovary, thyroid, and uterus. Fig. 2B shows the organ distribution of the 1,904 cases submitted from 2003 to 2014.

\section{Tissue of origin-specific diagnoses}

Among the 1,904 cases, the requests for 22 cases were not for a review of specific slides or pathologic reports. The contents of these consultations were questions about definition, biologic behaviors, or diagnostic methods for specific diseases. In 42 cases, submitters asked for a review of the cytopathologic diagnosis: two provided cervicovaginal smear slides, and 40 provided fineneedle aspiration slides of the thyroid. Thus, we excluded these 64 cases. In 37 of the remaining 1,840 cases, advisors could not provide a conclusive diagnosis because submitters did not provide appropriate information such as adequate pathology slides, slides with sufficient tissue, or complete clinical information. Finally, 1,803 cases were reviewed and classified based on the provided pathologic diagnosis. We analyzed these cases based on tissue of origin (Table 1, Electronic Supplementary Table S2-S21). For convenient presentation, we unified the diagnostic terms of neoplasms according to the most recently updated World Health Organization (WHO) classifications of tumors of each system.

\section{Colon and rectum}

The two main diagnoses in this category were "adenocarcinoma" and "neuroendocrine tumor (NET)," accounting for $42.5 \%$ 

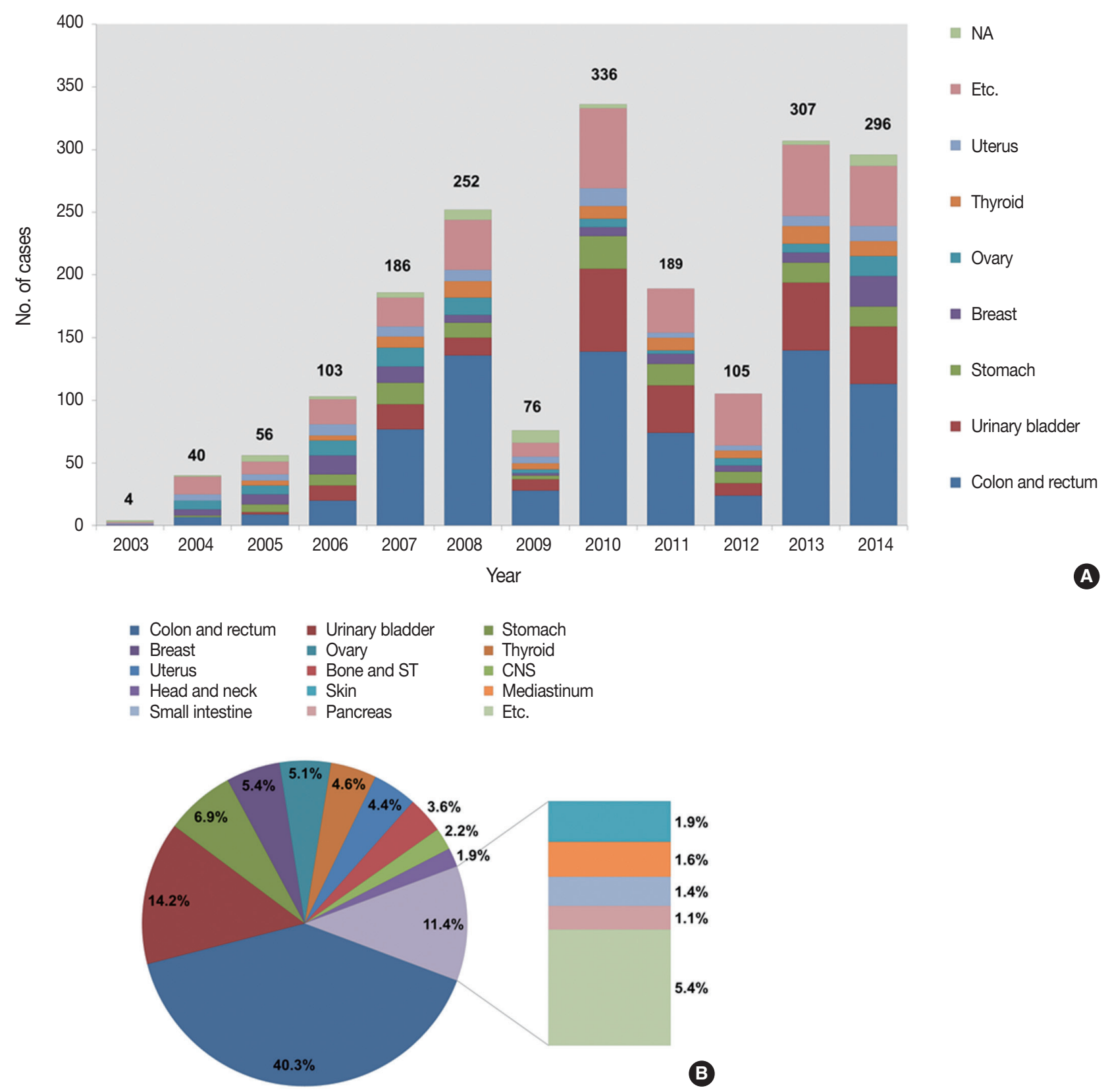

Fig. 2. Number of consultation cases from 2003 to 2014 and their tissues of origin. (A) The number of consultation cases has increased since 2003, although there are some variations. The colon and rectum have been the most common tissues of origin in recent years. (B) Of the entire set of consultation cases during the 12 study years, the colon and rectum were the most common tissues of origin, followed by the urinary bladder and stomach. NA, not applicable; ST, soft tissue; CNS, central nervous system.

(321 of 755) and $50.7 \%$ (383 of 755) of cases, respectively. Sixty-eight percent of the adenocarcinoma cases were "adenocarcinoma in situ" (263 of 321). The other 38.0\% were early-stage tumors with only mucosal or submucosal invasion. In terms of NET, $78.6 \%$ originated from the rectum (302 of 383).

\section{Urinary bladder}

The urinary bladder was the second most common tissue of origin. Most of the cases in this category were urothelial carcinoma (from non-invasive papillary urothelial carcinoma to invasive urothelial carcinoma). The single most common entity was "non-invasive papillary urothelial carcinoma, low grade," and it accounted for $70.7 \%$ (186 of 263) of the urinary bladder cases. The second-most common (29 of 263, 11.0\%) was "papillary urothelial neoplasm with low malignant potential." 
Table 1. Diagnoses of consultation cases of the three major tissues of origin: the colon and rectum, urinary bladder, and stomach

\begin{tabular}{|c|c|}
\hline Tissue of origin-specific diagnoses & No. of cases \\
\hline \multicolumn{2}{|l|}{ Colon and rectum } \\
\hline Hyperplastic polyp & 4 \\
\hline \multicolumn{2}{|l|}{ Tubular or tubulovillous adenoma } \\
\hline Low-grade dysplasia & 11 \\
\hline High-grade dysplasia & 28 \\
\hline \multicolumn{2}{|l|}{ Adenocarcinoma } \\
\hline Adenocarcinoma in situ & 263 \\
\hline Adenocarcinoma with submucosal invasion & 58 \\
\hline \multicolumn{2}{|l|}{ Neuroendocrine tumor } \\
\hline Colon & 81 \\
\hline Rectum & 302 \\
\hline \multicolumn{2}{|l|}{ Gastrointestinal stromal tumor } \\
\hline Low risk & 3 \\
\hline Intermediate risk & 1 \\
\hline Extranodal marginal zone lymphoma & 2 \\
\hline Chronic inflammation & 1 \\
\hline Total & 755 \\
\hline \multicolumn{2}{|l|}{ Urinary bladder ${ }^{a}$} \\
\hline \multicolumn{2}{|l|}{ Urothelial proliferative lesion } \\
\hline Urothelial hyperplasia & 1 \\
\hline Urothelial papilloma & 4 \\
\hline Urothelial papilloma with atypia & 2 \\
\hline Urothelial dysplasia & 1 \\
\hline PUNLMP & 29 \\
\hline Non-invasive papillary urothelial carcinoma, LG & 186 \\
\hline Non-invasive papillary urothelial carcinoma, $\mathrm{HG}$ & 8 \\
\hline Invasive urothelial carcinoma & 22 \\
\hline Urothelial carcinoma in situ & 4 \\
\hline Squamous cell carcinoma in situ & 1 \\
\hline Metastatic adenocarcinoma & 1 \\
\hline Cystitis & 4 \\
\hline Total & 263 \\
\hline \multicolumn{2}{|l|}{ Stomach } \\
\hline Tubular adenocarcinoma & 30 \\
\hline Signet ring cell carcinoma & 4 \\
\hline Adenocarcinoma in situ & 12 \\
\hline Tubular adenoma with high-grade dysplasia & 3 \\
\hline Tubular adenoma with low-grade dysplasia & 2 \\
\hline \multicolumn{2}{|l|}{ Gastrointestinal stromal tumor } \\
\hline Very low risk & 8 \\
\hline Low risk & 13 \\
\hline Intermediate risk & 9 \\
\hline High risk & 7 \\
\hline Unknown & 3 \\
\hline Extranodal marginal zone lymphoma of MALT & 10 \\
\hline Neuroendocrine tumor & 15 \\
\hline Granular cell tumor & 1 \\
\hline Leiomyoma & 2 \\
\hline Schwannoma & 1 \\
\hline Nonneoplastic polyp ${ }^{b}$ & 3 \\
\hline Chronic gastritis & 3 \\
\hline Total & 127 \\
\hline
\end{tabular}

PUNLMP, papillary urothelial neoplasm with low malignant potential; LG, low grade; HG, high grade; MALT, mucosa-associated lymphoid tissue. ${ }^{a}$ All diagnoses are based on the 2004 World Health Organization classification; 'Two fundic gland polyps and 1 hyperplastic polyp are included in this category.

\section{Stomach}

As a single entity, "adenocarcinoma" was the most common diagnosis of the cases in this category ( 46 of 127, 36.2\%). Adenocarcinoma in situ was also a frequent diagnosis (12 of 46, $26.1 \%$ ), followed by gastrointestinal stromal tumor (GIST) and NET (40 of $127,31.5 \%$ and 15 of $127,11.8 \%$, respectively). We were able to obtain the risk classification of 37 submitted GISTs based on the National Institutes of Health (NIH) consensus criteria, including tumor size and mitotic activity. ${ }^{6,7}$ Based on these criteria, tumors in the low risk group were the most common (13 of $40,32.5 \%)$.

\section{Major diagnoses from other tissues of origin}

Electronic Supplementary Table S2-S21 details the specific diagnoses of the remaining tissues of origin. For breast as the tissue of origin, the diagnoses varied from benign ductal epithelial lesions such as columnar cell change to invasive ductal carcinoma (Electronic Supplementary Table S2). As a single entity, phyllodes tumor comprised the largest portion of the category (30 of $100,30.0 \%$ ). Among these, borderline phyllodes tumor (19 of $30,63.3 \%$ ) based on the WHO classification was the most common diagnosis. ${ }^{8}$

Female genital tissues, the ovary and uterus, were also common tissues of origin. In the ovary, surface epithelial neoplasm was the most common lesion (Electronic Supplementary Table S3). Forty of 95 cases (42.1\%) were epithelial tumors, and half of these were borderline tumors. Granulosa cell tumor was also a common neoplasm (33 of 95, 34.7\%), with the ovary as the tissue of origin. In cases with the uterus as the tissue of origin, those of the cervix were slightly more common than those of the corpus (43 vs 33). Among cases with the cervix as the tissue of origin, squamous cell carcinoma in situ (14 of 43) and microinvasive squamous cell carcinoma (12 of 43) together accounted for $60.5 \%$ of cases. Among cases with the corpus as the tissue of origin, those of smooth muscle neoplasm were the most frequent. In particular, cases of smooth muscle neoplasm of uncertain malignant potential were more commonly submitted than other entities in this category (Electronic Supplementary Table S5).

The thyroid was the fifth most common tissue of origin, followed by the ovary. While the main intent of the consultation in cases with other organs as the tissue of origin was confirmation of the original diagnosis or classification of the disease based on classification system, the content of consultation in cases of thyroid origin was questions regarding methods for diagnosing papillary carcinoma. One of the main questions in these cases was whether a pathologist could provide a definite diagnosis of 
papillary carcinoma with only a fine-needle aspiration cytology (FNAC) specimen. This question was included in the consultation of 34 cases. In 15 of these 34 cases, the consulting pathologists answered that experienced pathologists could confirm the diagnosis of papillary carcinoma without additional histologic confirmation. In the other 19 cases, the consultants replied that additional histologic confirmation was needed to confirm the diagnosis because of the possible discrepancies between the cytology reports and the surgical pathology reports.

In other categories, most of the users provided surgical pathology slides. However, in the thyroid category, $46.0 \%$ of the users (40 of 87) provided cytology slides of FNAC specimens. According to the Bethesda system for reporting thyroid cytopathology, 31 of 40 cases belonged to category VI (malignant), and all of them were initially diagnosed as "papillary carcinoma." Nine of the 40 cases belonged to category V (suspicious for malignancy), and most of them were initially diagnosed as "suspicious for papillary carcinoma" (Electronic Supplementary Table S4).

Bone, soft tissue, and central nervous system were also common tissues of origin. In these categories, there was no predominant diagnosis, but a variety of rare tumors were submitted to confirm the original diagnosis (Electronic Supplementary Tables S6, S7). Most of the minor categories of tissues of origin such as head and neck, skin, kidney, and gallbladder included several minor diagnoses, without one predominant diagnosis. However, the mediastinum, small intestine, appendix, and pituitary gland had predominant diagnoses. Most of the cases with the mediastinum as the tissue of origin were thymomas (26 of $30,86.7 \%$ ), and "pituitary adenoma" was the only diagnosis in cases with the pituitary gland as the tissue of origin ( 5 of 5 , $100 \%$ ). In the small intestine category, GIST (13 of 24, 54.2\%) and NET ( 9 of $24,37.5 \%$ ) comprised most of the diagnoses. All submitted cases with the appendix as the tissue of origin were either NET ( 7 of $10,70.0 \%$ ) or low-grade appendiceal mucinous neoplasm (3 of 10, 30.0\%).

Across the entire dataset, regardless of tissue of origin, NET of the digestive system was the most common diagnosis. Four hundred nineteen of 1,803 cases $(23.2 \%)$ were NETs from various organs. Adenocarcinoma of the digestive system was the next most common diagnosis $(20.6 \%, 372$ of 1,803). Non-invasive papillary urothelial carcinoma from the urinary system was also frequently diagnosed, representing the third most frequent diagnosis and accounting for $20.0 \%$ (198 of 1,803) of the entire set of cases.

\section{DISCUSSION}

In Korea, most surgical pathology consultations are divided into two types. The first type is the so-called institutional pathology consultation or second opinion of pathologic slides. ${ }^{10}$ This type of consultation is common in cases where a pathological diagnosis is made at one hospital, and subsequent therapy is provided at another. In such a situation, the pathologists at the new hospital are usually asked to review the original pathologic reports and slides to confirm the diagnosis. Another type of consultation is usually requested by pathologists who encounter difficult cases. In such cases, Korean pathologists consult with other pathologists working in teaching hospitals. This type of consultation is usually termed as an extra-departmental pathology consultation or a personal consultation. ${ }^{11}$ The ultimate purpose of these consultations is to improve diagnostic accuracy and provide the best treatment to patients. In general, appropriate consultations are regarded as a helpful step for reducing errors in surgical pathology. ${ }^{12}$

This review of the 12-year MAS by the KSP revealed several important facts. First, we discovered the existence of another type of consultation for the accurate classification and coding of diseases for insurance reimbursement. Except for nine cases, all consultation cases in the current study were submitted by private health insurance companies or insurance adjustment companies who were hired by patients or insurance companies. The main purpose of these consultations seemed to be adjustment of insurance payments based on the new disease codes. Several studies have retrospectively reviewed pathologic consultations. However, those studies were examining institutional or personal pathologic consultations with a medical purpose. ${ }^{10,11,13-15}$ Most prior studies have focused on the quality of consultations and diagnostic discrepancy between the primary pathologist and consulting pathologist. ${ }^{10,11,14}$ Thus, this is the first study on pathologic consultation for the purpose of reimbursement of health insurance.

In Korea, all citizens have to acquire mandatory National Health Insurance. The public sector of National Health Insurance covers only part of the entire medical expenditure; therefore, most citizens purchase supplementary private health insurance. The National Statistical Office reported that more than $64 \%$ of individuals purchased private health insurance in 2010. As a result of this demand, the private health insurance market is growing rapidly. ${ }^{16}$ Conflicts about health insurance payments are also increasing. Both public and private forms of coverage for medical expense reimbursement are based on the disease clas- 
sification code assigned by clinicians based on the pathologic reports. ${ }^{17}$ Therefore, coding of tumors is an important issue in establishing insurance reimbursement. Therefore, pathologic consultation for insurance reimbursement purposes is expected to increase.

Second, certain types of tumors were frequently consulted. Regardless of the tissue of origin, NET of the digestive system was the most common diagnosis (419 of 1,803). The issue of staging and classification of NET is as yet unsettled..$^{18}$ In addition, the staging and classification systems of NET are various and complex. Many doctors still use different guidelines in classifying NETs, which might result in confusion in the clinical setting and coding. Han et al. ${ }^{17}$ used an internet-based survey and reported coding discrepancy among endoscopists when diagnosing NETs in the lower gastrointestinal tract. When given the same pathology report of a G1 NET of $1.5 \mathrm{~cm}$ size, with submucosal invasion, no lymphovascular invasion, a Ki-67 index less than $1 \%$, and a clear resection margin, $29.2 \%$ of endoscopists classified the tumor as malignant $(-/ 3), 61.5 \%$ classified it as having uncertain behavior (-/1), and $8.9 \%$ classified it as benign (-/0). Our study demonstrates that the disagreement of coding of tumors due to a lack of a unified and clear classification system could eventually affect insurance reimbursement and became a social issue.

Adenocarcinoma in situ of the colon and rectum and non-invasive urothelial carcinoma of the urinary bladder were two of the most common diagnoses in the consulted cases. In addition, the main request was determining the behavior codes of these tumors. In Korea, the KCD is the standard disease coding system and was established after the translation of the ICD by Statistics Korea to allow communication in a common language across clinical settings. ${ }^{5}$ The ICD-O is a fundamental classification system for tumors, the coding of which constitutes a dual classification system for both topography (site) and morphology (histology, behavior, and grading of malignancy). The ICD-O was originally developed for cancer registration, but it is also used by healthcare providers for quality control and by researchers

Table 2. Behavior codes of the International Classification of Diseases-Oncology-3 (ICD-O-3)

\begin{tabular}{ll}
$\begin{array}{ll}\text { ICD-O-3 } \\
\text { code }\end{array}$ & Disease \\
\hline$/ 0$ & Benign \\
$/ 1$ & Uncertain whether benign or malignant (borderline malignancy, \\
& low malignant potential, uncertain malignant potential) \\
$/ 2$ & Carcinoma in situ (intraepithelial, noninfiltrating, noninvasive) \\
/3 & Malignant, primary site \\
/6 & Malignant, metastatic or secondary site \\
\hline 9 & Malignant, uncertain primary or secondary site \\
\hline
\end{tabular}

for clinical trial recruitment, among other purposes. ${ }^{19}$ The revised ICD-O-3 added a last fifth digit that represents the biologic behavior of tumors (Table 2).

Traditionally, the behavioral nature of tumors is classified as malignant or benign. The two most important characteristics of a malignant tumor are local invasion and distant metastasis. In actual practice, the diagnosis and classification of tumors are not that simple. Neoplasms with uncertain behavior (-/1) or carcinoma in situ (-/2) also exist. Some malignant tumors can evolve from a pre-invasive stage referred to as carcinoma in situ, which means that the cancer cells display the cytologic features of malignancy without invasion of the basement membrane. ${ }^{20}$ Adenocarcinoma in situ and non-invasive urothelial carcinoma are such examples. The -/2 (in situ) tumors show more favorable behavior than the $-/ 3$ (malignant) tumors. Based on this finding, private health insurances usually only reimburse $10 \%$ to $20 \%$ of the amount of reimbursement to patients with -13 (malignant) tumors. However, the underlying concepts of these tumors cannot be easily understood by patients because -/2 (in situ) tumors are also referred to as "cancer" in general. Thus, in this kind of situation, patients should perhaps seek medical or legal advice to ensure that they are reimbursed correctly.

The ICD-O is a useful system for the purposes of an internationally unified principal of disease coding. However, it is difficult to ensure that doctors assign an identical code to the same tumor. This seems to be due to numerous co-existing classification systems and synonyms for the same tumor, and doctors have their own classification preferences. Physicians also have their own viewpoints on the prognosis and biologic behavior of tumors based on experiences, and research, but these viewpoints could be changed. New forms of tumors and new opinions about tumor behavior are continuously being proposed. Because the ICD-O-3 was created for the statistical analysis of tumor prevalence and death rates, it cannot satisfy all the various viewpoints of doctors. Sometimes it makes doctors confused when they give a code to the disease and causes coding discrepancies.

These coding discrepancies between doctors might give rise to conflicts between patients and health insurance providers. Even with the same condition, patients can receive different payments from insurance providers depending upon the code chosen by the clinician or that of the pathologist reporting the diagnosis. Many patients and private health insurance providers recognize the possibility of discrepancies and therefore use advisory services such as the KSP or individual pathologists to obtain a more profitable diagnosis or classification code. We expect that this kind of situation will increase as the private health in- 
surance market expands.

Through this review of 12 years of MAS provided by the KSP, we have recognized that consultations associated with reimbursement of private health insurance account for a large proportion of pathologic advisory services, and that the coding of tumors is an important issue in Korean society. In particular, the complex coding systems and coding discrepancies among clinicians and/or pathologists are problems that need to be solved. The best solution is to establish a better tumor classification and coding system that is able to reflect the biologic nature of tumors and is easily understood by non-experts.

In order to accomplish this, pathologists must play a role, because the classification of tumors should be based on cytopathologic characteristics that best reflect the biologic nature of tumors. Korean pathologists have been actively working toward this goal. In collaboration with the National Cancer Center, the KSP has participated in the confirmation of diagnostic terms; standardization of diagnostic formats; and clarification and assessment of multiple primaries, primary sites, ICD-O codes, and education of pathologists. ${ }^{21}$ Several study groups of the KSP have proposed behavior codes for several tumors with controversies regarding classification and coding. ${ }^{21-23}$

Thanks to these efforts, there were only a few discrepancies in coding of the same tumor among different subspecialty pathologists in this review (data not shown). However, a small proportion of coding discrepancies existed in certain tumors such as NET and granulosa cell tumor of the ovary. These cases were mostly diagnosed and consulted before the unified guidelines were proposed by the KSP. We therefore presume that the effort of the KSP in proposing and presenting a simplified and unified classification and coding system is having a positive effect. Nevertheless, this review of the MAS of the KSP reveals that there are still problems with the classification and coding systems for neoplasms, and that they will continue to be important issues. Therefore, we should persist in our efforts to focus attention on and further improve these areas.

\section{Electronic Supplementary Material}

Supplementary materials are available at Journal of Pathology and Translational Medicine (http://jpatholtm.org).

\section{Conflicts of Interest}

No potential conflict of interest relevant to this article was reported.

\section{Acknowledgments}

We would like to thank Kum Mi Kim, General Manager of the Korean Society of Pathologists, and Min Sun Kim, Manager of the Korean Society of Pathologists, for collecting and managing the consulting data and providing secretarial assistance.

\section{REFERENCES}

1. Recommendations of the Association of Directors of Anatomic and Surgical Pathology. Part II. Consultations in surgical pathology. Hum Pathol 1993; 24: 691-2.

2. Simpson PR, Tschang TP. ADASP recommendations: consultations in surgical pathology. Association of Directors of Anatomic and Surgical Pathology. Hum Pathol 1993; 24: 1382.

3. Consultations in surgical pathology. Association of Directors of Anatomic and Surgical Pathology. Am J Clin Pathol 1994; 102: 152-3.

4. Fritz A, Percy C, Jack A, et al. ICD-O: International classification of diseases for oncology. 3rd ed. Geneva: World Health Organization, 2000.

5. Statistics Korea. Introduction to the classification of diseases [Internet]. Daejeon: Statistics Korea, [cited 2015 Oct 1]. Available from: https://kssc.kostat.go.kr.

6. Recommendations on quality control and quality assurance in anatomic pathology. Association of Directors of Anatomic and Surgical Pathology. Am J Surg Pathol 1991; 15: 1007-9.

7. Agaimy A. Gastrointestinal stromal tumors (GIST) from risk stratification systems to the new TNM proposal: more questions than answers? A review emphasizing the need for a standardized GIST reporting. Int J Clin Exp Pathol 2010; 3: 461-71.

8. Lakhani SR, Ellis IO, Schnitt SJ, Tan PH, van de Vijver MJ. WHO classification of tumours of the breast. Lyon: IARC Press, 2012.

9. Cibas ES, Ali SZ; NCI Thyroid FNA State of the Science Conference. The Bethesda System For Reporting Thyroid Cytopathology. Am J Clin Pathol 2009; 132: 658-65.

10. Tsung JS. Institutional pathology consultation. Am J Surg Pathol 2004; 28: 399-402.

11. Hsu CY, Su IJ, Lin MC, Kuo TT, Jung SM, Ho DM. Extra-departmental anatomic pathology expert consultation inTaiwan: a research grant supported 4-year experience. J Surg Oncol 2010; 101: 430-5.

12. Abt AB, Abt LG, Olt GJ. The effect of interinstitution anatomic pathology consultation on patient care. Arch Pathol Lab Med 1995; 119: 514-7.

13. Azam M, Nakhleh RE. Surgical pathology extradepartmental consultation practices. Arch Pathol Lab Med 2002; 126: 405-12.

14. Renshaw AA, Pinnar NE, Jiroutek MR, Young ML. Quantifying 
the value of in-house consultation in surgical pathology. Am J Clin Pathol 2002; 117: 751-4.

15. Lipp ES, Clark AC, McLendon RE. Consultative issues in surgical neuropathology: a retrospective review of the rationale for submitting cases for expert review. Am J Clin Pathol 2015; 143: 807-11.

16. Jeon B, Kwon S. Effect of private health insurance on health care utilization in a universal public insurance system: a case of South Korea. Health Policy 2013; 113: 69-76.

17. Han DS, Sohn JH, Byeon JS, Choi H, Kim JM. Diagnostic coding for intramucosal carcinoma and neuroendocrine tumor in the colorectum: proposal for avoiding confusing coding in Korea. Clin Endosc 2015; 48: 216-20.

18. Bosman FT, Carneiro F, Hruban RH, Theise ND. WHO classification of tumours of the digestive system. 4th ed. Lyon: IARC Press, 2010.
19. Kavuluru R, Hands I, Durbin EB, Witt L. Automatic extraction of ICD-O-3 primary sites from cancer pathology reports. AMIA Jt Summits Transl Sci Proc 2013; 2013: 112-6.

20. Kumar V, Abbas AK, Aster JC, Fausto N. Robbins and Cotran's pathologic basis of disease. 8th ed. Philadelphia: Saunders, 2010.

21. Sohn JH, Gong G, Kim KR, et al. Proposal for creating a guideline for cancer registration of microinvasive tumors of the breast and ovary (II). Korean J Pathol 2012; 46: 226-32.

22. Yoo C, Kang CS, Choi YL, et al. A proposal for creating a guideline for cancer registration of the fibromatosis, PEComa group, malignant lymphoma in situ and dendritic cell tumors (III). Korean J Pathol 2012; 46: 436-42.

23. Jung ES, Kang YK, Cho MY, et al. Update on the proposal for creating a guideline for cancer registration of the gastrointestinal tumors (I-2). Korean J Pathol 2012; 46: 443-53. 